\title{
Neurosurgery for mental disorder: past and present
}

\author{
George W. Fenton
}

\section{The birth of psychosurgery}

Carlyle Jacobsen and John Fulton, at Yale in the early and mid-1930s, investigated primate frontal lobe function. Following bilateral frontal lobecotomy, monkeys and chimpanzees developed a delayedresponse deficit, being unable to perform tasks when there was a significant delay between the stimulus and the required response. There were accompanying behavioural changes, with the animals becoming more placid and lethargic and losing their characteristic inquisitive initiative. They became distractible, with difficulty in maintaining an attention set. They had great difficulty in adapting to changes in the directions of an already learned task, tending to perseverate.

Fulton and Jacobsen did experiments on two chimpanzees, Becky and Lucie, who were laboratory favourites. Lucie performed well on the delayedresponse tasks prior to surgery but afterwards made many errors and displayed frequent temper tantrums with the consequent delays in food reward. In contrast, Becky exhibited a 'neurotic' behaviour pattern during baseline testing, with temper tantrums, throwing herself on the floor, defaecating and urinating. Following surgery, she was calm and participated in the delayed-response paradigm without difficulty. She made many errors that she was oblivious to.

The data from the experiments with Lucie and Becky were presented at a symposium on frontal lobe function during the Second World Congress of Neurology, held in London in July 1935. Attending the symposium was the Professor of Neurology from
Lisbon, Egas Moniz - formerly Portuguese Foreign Minister. He already had an international reputation for developing cerebral arteriography between 1927 and 1935 . He was immediately struck by the calming effect on Becky of the frontal lobectomy, and speculated about its possible application to the treatment of intractibly ill, mentally disordered patients. He had already been considering the feasibility of interrupting the frontal lobe projections as a potential surgical treatment for intractible mental disorder for several years. (The influence of Jacobson and Fulton's work on Moniz's ideas is now the subject of historical controversy (Pressman 1998; Berrios, 1997). There seems little doubt that he had been considering frontal lobe surgery for some years prior to the London Congress, although in retrospect, Moniz acknowledged the contributions of Jacobsen and Fulton's findings.)

On return to Lisbon after the congress, he collaborated with Almeida Lima, a young neurosurgeon. They carried out the first psychosurgery operations on 12 November 1935 . They operated on four patients - two with chronic depression and two with paranoid schizophrenia - using alcohol injections into the depths of the frontal white matter on each side. They later used an instrument, known as a leucotome, with a steel loop to crush the white matter. Finally, they adopted a leucotome with a steel band that cut the white matter. Within the first year, they had completed 20 operations on patients with intractible mental disorder of varied symptomatology. The results were published widely. Seven patients were considered 'cured', seven significantly improved, and six unchanged (Moniz, 1936) . Moniz had difficulty in recruiting further patients since the local psychiatric establishment

George Fenton is Emeritus Professor at the University of Dundee (Department of Psychiatry, Ninewells Hospital and Medical School, Dundee DD1 9SY), having been Head of the University Department of Psychiatry there (1983-1996), and formerly Professor and Head of the Department of Mental Health, Queen's University of Belfast (1976-1983). Previously a consultant psychiatrist and neurophysiologist at the Maudsley Hospital and a senior lecturer at the Institute of Psychiatry, University of London, his special interests include neuropsychiatry, clinical neurophysiology and neurosurgery for mental disorder. 
did not approve. Nevertheless, he was awarded the Nobel prize for Medicine and Physiology in 1949, jointly with Walter Hess, the neurophysiologist.

With all the publicity given to Moniz's work and the desperate need to find more effective treatment for the thousands of mentally disordered patients institutionalised in large mental hospitals, it is hardly surprising that prefrontal leucotomy was practised widely throughout the developing world. By 1940 , at least 500 operations were carried out worldwide, with published series from Italy, Romania, Brazil, France, and the USA.

Excellent reviews of the early days of psychosurgery are given by Shutts (1982), Swayze (1995), Berrios (1997) and Pressman (1998). See Box 1 for an outline of the historical developement.

\section{The lobotomy era}

Walter Freeman, a neuropathologist and neurologist, who had met Moniz at the London Congress, was particularly active in the USA. He collaborated with James Watts, a neurosurgeon who had previously done experimental neurophysiology with Fulton. In Washington they developed the standard prefrontal lobotomy. In this operation a burr hole was made in each temporal region though which a leucotomy knife was swept up and down in an arc, severing the frontal cortical-subcortical connecting white matter and making large lesions of variable size (Freeman et al, 1950). The blind 'freehand' operation and its many modifications were used over the next 20 years. The transorbital leucotomy technique developed by Freeman was widely used and particularly controversial. This psychosurgery variant did not require neurosurgical skill and involved penetrating the roof of each orbit with a sharp instrument like an ice pick, angled upwards and backwards and rotated laterally on each side to make the cut. Freeman used to anaesthetise the patients with electroconvulsive therapy prior to carrying out the operation in a range of non-surgical settings. The transorbital leucotomy approach horrified neurosurgeons because it was not carried out under sterile conditions in an operating theatre and was often performed by people without surgical training, ill-equipped to cope with such acute complications as haemorrhage. Despite these concerns, complications were relatively rare and the prevalence of severe personality changes less than those following the standard leucotomy procedure. Hence, the transorbital approach became widely used.

\section{Leucotomy in Britain}

The first operations in Britain were carried out in Bristol in 1940 by Wilfred Willway, surgical registrar at the Bristol Royal Infirmary, with the encouragement and guidance of Professor Golla of the Burden Neurological Institute. Drs Hutton, Fleming and Fox reported the results of the first eight patients in the Lancet in 1941 (Hutton et al, 1941). The same issue included a cautious but supportive editorial (Anonymous, 1941). The Bristol group continued to be active, Hutton reporting on 50 cases in 1943 (Hutton, 1943), while Fleming, one of her previous collaborators, teamed up with Wylie McKissock to publish in the same issue of the Lancet details of a further 15 cases, mainly melancholic in nature (Fleming \& McKissock, 1943). The latter went on to perform more than 1400 leucotomies. These early studies provided the impetus for the widespread use of prefrontal leucotomy. Between 1948 and 1954 around 1100 operations were carried out each year in England and Wales. Even in the early days their use was a source of controversy since mind-altering surgery that achieved its therapeutic aim by damaging apparently healthy brain was regarded as unethical by many psychiatrists and members of the general public. There were also scientific concerns about the validity of the claims for efficacy and the risks of frontal lobe mental and behavioural deficits.

The operative mortality was $2.7 \%$, most deaths being due to cerebral haemorrhage. Twenty-nine per cent developed postoperative epilepsy although seizures were infrequent and easy to control by medication. More serious was the $3 \%$ prevalence of severe frontal lobe syndrome and less extreme but significant personality deficits in most patients. These adverse complications greatly added to the controversy about the ethics of such surgery. The cavalier attitudes of many of its early practitioners towards selection and assessment of patients and uncritical claims about efficacy also added fuel to the storm of criticism.

What has tended to be overshadowed by the ethical debate concerning the early operations is their relative clinical efficacy in the era before effective psychotropic medication. In a review of 10365 patients operated on in England and Wales between 1942 and $1954,41 \%$ were recovered or greatly improved, $28 \%$ minimally improved, $25 \%$ unchanged, $2 \%$ worse and $4 \%$ died (Tooth \& Newton, 1961). Two-thirds of those operated on had a diagnosis of schizophrenia and one-third affective disorder. Sixty-three per cent of the latter were recovered or greatly improved, compared with $30 \%$ of the former. 


\section{Modified operations}

The next step in the evolution of psychosurgery was made possible by Fulton (1951). He carefully analysed the extent and site of the lesions at postmortem examination. By relating the latter findings to clinical outcome, he was able to demonstrate that lesions confined to ventromedial quadrants of the frontal lobes gave the best clinical results with the lowest risk of serious personality change. This led to open brain surgery with the lesions restricted to the ventromedial quadrants of the frontal lobe or related areas. The most common modified operations were the rostral leucotomy first carried out by Wylie McKissock in 1948, orbital undercutting introduced by Scoville in the USA in 1949 and modified by Knight in England in 1950, and the bimedial leucotomy carried out by Peter Schurr for 20 years. Cingulectomy (bilateral excision of Brodmann's area 24) was also introduced in the late-1940s in the USA and carried out in Oxford by Cairns and colleagues. During the mid- and late-1950s the modified operations gradually replaced the standard leucotomy, so that by $1961,80 \%$ of the 447 leucotomy operations carried out in England and Wales were modified in nature.

\section{Decline in leucotomy}

By the mid-1950s the introduction of antipsychotic drugs provided an effective medical treatment for the positive symptoms of schizophrenia, and psychosurgery was no longer an indication in this

Box 1. History of neurosurgery for mental disorder

First leucotomy operation performed by Moniz and Lima in November 1935

Standard prefrontal leucotomy operation pioneered by Freeman and Watts in Washington, DC in 1936

First operations in Britain in Bristol, 1940

Modified freehand leucotomies with cuts restricted to ventromedial frontal lobe quandrants, developed in late 1940s and early 1950 s

Stereotactic neurosurgery for mental disorder first performed in 1960s condition. The addition of effective antidepressant medication in the late 1950s also had a significant impact on referral for leucotomy. Follow-up studies of both the standard and restricted lesion operations had shown that patients with intractable depressive, anxiety or obsessive-compulsive disorders had a consistently better outcome than patients with schizophrenia. As a consequence, the selection criteria narrowed to focus mainly on patients with these former disorders. By 1961, the numbers of patients operated upon in England and Wales had fallen by more than half, compared with the numbers operated on in 1954. This trend has continued ever since. By 1979, the annual number of operations had fallen to 70 , with a further decline to 21 by 1985 .

\section{Stereotactic neurosurgery}

Even the modified operations did not solve the adverse effects problem. For example, although rostral leucotomy produced greater clinical improvement than the standard operation, one-third of patients showed a noticeable and often persistent reduction in drive and almost half were less restrained in speech and temper (Pippard, 1955). In a careful three-year follow-up of Maudsley Hospital patients, $69 \%$ displayed sustained improvement, but undesirable effects on personality occurred in $59 \%$ and were troublesome in $21 \%$ (Post et al, 1968).

The advent of stereotactic neurosurgery provided neurosurgeons with the capacity to make accurately localised lesions. Since the early 1960 s a series of stereotactic operations have been developed (see Box 2). There is no consensus on the optimum target site for the operation but the aim in most procedures is to interrupt the limbic system circuits at a convenient site, usually in the orbitomedial frontal cortex, anterior limbs of the internal capsule or cingulate areas.

Box 2. Targets for stereotactic neurosurgery for mental disorder

Beneath the head of each caudate nucleus: subcaudate tractotomy

Anterior limbs of both internal capsules: bilateral anterior capsulotomy

Combined lesions in orbitomedial quadrants of each frontal lobe and cingulate areas on each side: limbic leucotomy

Cingulate areas on each side: cingulotomy 
Geoffrey Knight pioneered the stereotactic subcaudate tractotomy (SST) operation to simulate the open freehand orbital undercut procedure which had to be abandoned because of a high prevalence of epilepsy. In the initial SST operation, destructive lesions were made in the posterior part of the substantia innominata beneath the head of each caudate nucleus area using radioactivity from arrays of implanted ceramic rods containing a radioisotope of yttrium ( $\left.{ }^{90} \mathrm{Y}\right)$, which has a half-life of about 68 hours. This proved a safe technique and well over 1000 operations have been performed in London by Knight and his successors (Bridges et al, 1994). Since ${ }^{90} \mathrm{Y}$ is no longer readily available, similar lesions in the same sites are now made by electrocoagulation.

In Sweden, the anterior limbs of each internal capsule have been targeted, an operation known as a bilateral anterior capsulotomy (Mindus et al, 1994). The lesions are usually thermal, although a gamma knife technique is now being pioneered in Sweden and the USA. In contrast, the anterior cingulotomy operation targets the cingulate tracts on each side (Baer et al, 1995). Finally, the limbic leucotomy procedure involves lesions in both the orbitomedial quadrants of each frontal lobe and the cingulate areas on each side (Kitchen, 1995). It has been suggested that depression is best treated by lesions in the basomedial frontal region (i.e. subcaudate tractotomy or cingulotomy), whereas obsessivecompulsive disorder (OCD) responds best to capsulotomy operations. More rigorous evidence is required to substantiate this claim. Studies directly comparing the effect of the different target sites on clinical outcome have yet to be carried out.

\section{Controversial issues}

Psychosurgery has been subject to controversy since its inception (see Box 3 ), with intertwining concerns about the ethics of such brain-damaging surgery, the problems of informed consent, the potential requirement for external regulation and the need for scientific rigor in case selection and assessment of outcome.

Many opponents of neurosurgery for mental disorder argue that an operation that damages the brain to achieve its therapeutic effect is unethical in principle. The more extreme advocates of this view present as supportive evidence the damaging effects on personality of the old standard prefrontal leucotomy and tend not to discriminate between the latter operation and the new stereotactic methods that cause carefully targeted and localised damage with a low incidence of adverse personality change. Doubtless such arguments will only abate when techniques are developed that do not directly damage brain tissue, such as chronic stimulation through implanted electrodes. In practice, careful consideration of each patient as an individual, balancing the risk of adverse effects - now relatively low - against the possibility of significant improvement, is preferable to abstract ideological argument.

Another concern of opponents of neurosurgery for mental disorder is its potential to be misused as a method of social control - to control awkward or socially disapproved behaviour in the absence of psychopathology. The current policy of narrowing selection to a small number of well-defined categories of treatment-refractory mental disorder, coupled with external peer review, makes such misuse virtually impossible. Furthermore, this issue was examined by the 1977 US Department of Health, Education and Welfare Commission on Psychosurgery (National Commission for the Protection of Human Subjects of Biomedical and Behavioral Research, 1977) and was found to have no substance.

The lack of a precise neural model of how neurosurgery for mental disorder works remains a valid concern, which many non-medical people bring up when the subject is discussed, and is a challenge to neuroscientists to improve our knowledge of the brain mechanisms that regulate mood. Psychiatrists live more comfortably with this uncertainty since many effective treatments are used long before the mechanism of action is understood.

A common objection to psychosurgery is the view that patients are operated upon as an easy alternative to psychotherapy. In reality, most patients selected for psychosurgery have already had a wide range of treatments including some form of psychotherapy, and have proved treatment-resistant. Indeed, an important selection criterion is that the disorder is demonstrated to be treatment-refractory according to a standard protocol of appropriate therapies, both physical and psychological.

The ability to give informed consent requires the patient to have adequate knowledge of the nature of

\section{Box 3. Controversial issues}

Ethics of brain-damaging surgery and potential for adverse effects on personality

Potential as method of social control

Lack of precise method of action

Neurosurgery as easy alternative to psychotherapy

Ability to give informed consent

Regulation by peer review

Problem of determining efficacy 
the surgery, its rationale, pros and cons, the common complications and the range of possible outcomes. It is important to ensure that the patient's autonomy is intact with no undue pressure from relatives, carers, or indeed physicians. Finally, one needs to check that consent is not a consequence of the patient's psychopathology, for example, a desire for punishment in a severely depressed patient with feelings of guilt or unworthiness. Despite this list of possible difficulties, problems with capacity for giving informed consent are relatively rare.

The demand for an independent, multi-disciplinary peer review process arose because of fears that patients may be 'rail-roaded' into psychosurgery by enthusiastic psychiatrists with a cavalier approach to clinical diagnosis and unrealistic and uncritical convictions about the efficacy of the surgery. Some psychiatrists and surgeons involved in neurosurgery for mental disorder have argued that such peer review, particularly if it is compulsory through statute, discriminates against mentally ill patients by depriving them of their autonomy. Patients with organic brain disorders, such as parkinsonism, are considered capable of consenting to surgery in the absence of special safeguards, even though mood disorder and communication difficulties are not uncommon features of their condition. However, the argument for external peer review has prevailed. Independent, multi-disciplinary peer review systems are now regarded as an essential part of psychosurgery programmes, their role being to check that the selection criteria have been met, to assess capacity for informed consent and to give a second opinion about predicted response to surgery. Such a process reassures the patients, their families and the general public that the selection for neurosurgery for mental disorder is being conducted with care and expertise, and protects the neurosurgery for mental disorder team from the risk of frivilous allegations of malpractice by zealous opponents.

The final area of contraversy is the problem of establishing clinical efficacy, which has dogged psychosurgery since the early days. This difficult issue will be discussed below.

\section{What's in a name?}

Having outlined the evolution of surgery for mental disorder from the standard prefrontal leucotomy to modern stereotactic neurosurgery, it is perhaps appropriate to discuss briefly nomenclature. Moniz coined the term 'prefrontal leucotomy' to describe the operation he pioneered. The word 'lobotomy' was preferred to 'leucotomy' in the USA. This difference in terminology between the USA and
Europe continued over the years in medical circles. However, lobotomy has become popular with the news media on both sides of the Atlantic as 'shorthand' to describe all neurosurgical operations for mental disorder. No distinction is made between the outdated prefrontal leucotomy operations and the quite different stereotactic procedures. 'Lobotomy' makes a better headline. Indeed, it is often used as a perjorative term by the more extreme, non-medical opponents of surgery.

The term 'psychosurgery' was first introduced by Moniz and has been defined by the World Health Organization (1976) as "selective surgical removal or destruction of nerve pathways for the purposes of influencing behaviour". This definition is too broad and includes stereotactic operations targeting the amygdala, the hypothalamus or the stria terminalis for aggressive behaviour, which are no longer performed. It is best to restrict the term psychosurgery to the treatment for identifiable psychiatric disorders by neurosurgical methods that produce the destruction and division of certain cerebral structures. This reflects current practice. The term 'neurosurgery for mental disorder' was introduced by the Scottish Office Working Group in 1996 (CRAG Working Group on Mental Illness, 1996) in order to focus on definable mental disorders and to eliminate the use of such outdated terms as lobotomy and leucotomy, which are not appropriate descriptions of the current stereotactic operations targeting the limbic circuits. A similar US term is 'functional neurosurgery for psychiatric disorder'.

\section{Efficacy of stereotactic neurosurgery}

The clinical efficacy of neurosurgery for mental disorder is difficult to evaluate. Ethical issues make randomised controlled trials difficult, if not impossible, to organise. The various centres use different stereotactic targeted lesions and there are no comparative studies examining the relative efficacies of the various techniques. The distribution of the clinical diagnoses of the patients operated upon varies between centres, as well as the nature and scientific rigour of assessment instruments, making comparison difficult. Long-term outcome studies (with postoperative follow-up periods of 10 years or more) contain small sample sizes. Clinical outcome is rarely assessed by raters independent of the neurosurgery for mental disorder team.

Nevertheless, there are now a number of welldocumented published studies from centres in Sweden, the UK, the USA and Australia using 
reliable and valid measures of mental state and behaviour. In several, comparison with small samples of age- and gender-matched patients suffering from the same disorder but not operated upon have been possible. A literature review on clinical effectiveness is published in the Scottish Office report (CRAG Working Group on Mental Illness, 1996). For both major depressive and obsessive-compulsive disorders, between one-third and two-thirds of patients are in the Pippard rating scale $A$ and $B$ categories (recovered; much improved or showing considerable symptom alleviation). Longer-term studies tend to have improvement rates at the lower end of this range. The median percentage of patients showing significant improvement is around $50 \%$. An exception is the Bristol study of 142 OCD patients followed up for 15-20 years (Bird \& Crow, 1990). Sixty-eight per cent showed complete recovery or marked improvement. Seventy-two gold electrodes were implanted stereotactically in the orbital and paracingulate areas of the frontal lobe and left in place for up to 10 months. Electrical stimulation and subsequently electrocoagulation was progressively carried out until the optimum response was reached. These procedures were performed during a prolonged in-patient stay with intensive psychological support and behavioural therapy, which may have contributed to the impressive outcome. This operation is no longer carried out. It was the brainchild of Harry Crow, neuropsychiatrist at the Burden Neurological Hospital, and did not outlast his retirement (Crow, 1961). In any event, in-patient stays of up to 10 months at a time do not fit in with the aegis of the new National Health Service.

Another observation that supports the relatively favourable follow-up study data is comparisons with the suicide rates following neurosurgery. Compared to the $15 \%$ suicide rate for non-surgically treated patients with chronic major depressive disorders, the suicide rates in the years following neurosurgery are much lower; $1 \%$ for SST, $5 \%$ for limbic leucotomy, $9 \%$ for cingulotomy. The total lack of suicides following capsulotomy may relate to the fact that capsulotomy has been used more often for OCD patients than for depressed patients. (See Box 4 for summary.)

Nevertheless, for the above-mentioned reasons, the follow-up outcome data remain unsatisfactory, and open to challenge from sceptics. Snaith (1994), in his editorial calling for better evaluation of neurosurgery for mental disorder, suggested five requirements for such an assessment:

(a) Independent assessment by mental health workers who had no role in the decisionmaking and who have different professional backgrounds; through audiotaping or video- taping of interviews before surgery and at follow-up for further independent evaluation.

(b) Clear pre-operative and postoperative information, and psychometric tests assessing aspects of both symptom severity and cognitive function.

(c) An adequate period following the intervention of at least a year before follow-up.

(d) Information from brain imaging before and after surgery in order to establish the location and extent of the stereotactic lesions. The fact that different centres use different stereotactic techniques adds to the confusion. However, if the various centres can be persuaded to use a common pre- and postoperative assessment protocol, then it should be possible to do comparative studies examining the efficacy of different techniques.

(e) A complete sample of patients, not just those who are willing and able to travel to take part in a clinic-based follow-up. This requires home-based evaluation.

\section{Complications}

\section{Acute complications of stereotactic surgery}

All these operations have in common an operative mortality of less than $0.1 \%$ and a low prevalence of immediate and long-term adverse effects. Like all operations on the brain, haemorrhage and/or infection are acute risks with hemiplegia in less than $0.3 \%$. More benign postoperative complications are transient confusion, lethargy and incontinence of urine which may persist for a few days or weeks post operatively. Increased sensitivity to medication following surgery seems to contribute to the onset

Box 4. Assessment of efficacy of stereotactic surgery for mental disorder

Essentially similar outcome rates regardless of site of stereotactic target

Median improvement rates of $50 \%$ across series, varying from $1 / 3$ to $2 / 3$ of patients; long-term outcome studies tend to show improvement rates at lower end of this range

Reduced suicide rates in the long-term Significant personality change rare 
and maintenance of postoperative confusion. Transient postoperative oedema is another factor.

\section{Longer-term complications}

In the longer-term, epilepsy is a significant complication; between $1 \%$ and $2 \%$ for the SST and capsulotomy operations, and a higher prevalence of $9 \%$ for the cingulotomy procedure. The seizures are usually few in number, infrequent in recurrence and responsive to antiepileptic medication. The frontal lobe syndrome is a very rare but not unknown complication of the stereotactic operations (Hussain et al, 1988; Hay et al, 1993).

Personality trait changes such as 'outspokenness', disinhibition, irritability, lack of consideration and lack of initiative, have been described after surgery. None has been regarded as socially incapacitating and some indicate changes in psychosocial adjustment rather than organic deficits. Some reflect mental state improvements following surgery, for example, improved mood leading to more assertiveness and less dependence. Others mark a return to premorbid functioning styles. Indeed, the only three long-term systematic studies of personality change after surgery report that in the majority of patients there was no significant negative impact on personality. In fact, there were improvements in mood, depth of feeling, anxiety proneness, obsessionality, sociability and level of dependency (Mindus \& Nyman, 1991; Sachdev \& Hay, 1995; Mindus et al, 1999).

In particular, there were no significant changes in measures of impulsivity or hostility. A 1988 review of 854 stereotactic operations reported marked personality change in $0.4 \%$ and mild personality change in 3\% (Kiloh et al, 1988).

General intelligence, cognitive processing speed, attention and memory show no long-term deficits, although in one of the long-term studies, OCD patients operated on had a significantly poorer performance on the Wisconsin Card Sorting Test, a test of frontal lobe functioning (Hay et al, 1993). In contrast, 23 depressed patients who underwent stereotactic subcaudate tractotomy showed no deficits in frontal lobe functioning (Kartsousis et al, 1991).

\section{Contemporary indications}

The main contemporary indications for stereotactic neurosurgery (see Box 5) are chronic treatment refractory major depressive disorder and OCD. Despite advances in the medical and psychological therapies for these conditions, there seems to be a residual number of treatment-resistant patients for whom neurosurgery is an appropriate treatment option when other potentially effective therapeutic strategies have failed. A 1994 survey of Scottish psychiatrists covering a population of 5 million estimated 195 such patients seen over the previous five years, at least 10 times greater than the number actually assessed. In the UK as a whole, the number of operations has averaged 23 a year in the period $1990-1994$, over $70 \%$ having been SST procedures performed at the Geoffrey Knight unit in London, now relocated from the Brook to Kings College Hospital. Other UK centres are Cardiff, Leeds, St George's Hospital London, and Dundee, which is the national Scottish centre.

\section{Who should carry out neurosurgery for mental disorder?}

Neurosurgery for mental disorder is best carried out in specialist centres where a multi-disciplinary team of psychiatrists, a neurosurgeon and a clinical psychologist with a special interest in these disorders, can work together to carry out the assessment of suitability for this type of surgery the pre-operative assessments, the surgery itself and subsequent follow-up (see Box 6).

The selection for surgery requires confirmation of the diagnosis, the establishment of chronicity (five years or more continuous illness) and the treatmentrefractory nature of the disorder according to a standard protocol of available treatments, both physical and psychological. The patient, their nextof-kin and carers need to be fully informed about the advantages and disadvantages of surgery by one or more members of the multi-disciplinary team. They should be given the opportunity to discuss these issues not only with members of the multidisciplinary team, but also with their friends and usual carers. Communication can be facilitated with the use of an information sheet written in simple

Box 5. Indications for neurosurgery for mental disorder

Chronic treatment refractory major depressive disorder

Chronic treatment refractory obsessivecompulsive disorder 
language describing the nature of the operation, the reasons for using it, possible complications and predicted outcome. Preparing information in this way facilitates the development of informed consent. Once such initial consent is obtained, it is necessary to involve the statutory bodies that deal with the protection of patients' interests - the Mental Health Act Commission in England and the Mental Welfare Commission in Scotland. Their consent is mandatory in England. In Scotland the Mental Welfare Commission is required by law to give consent in a case of detained patients only, but an informal arrangement exists for them to assess other patients and give their opinion about suitability for surgery.

A number of baseline assessments of mental state, social adjustment, personality, quality of life and cognitive function are required before the operation, with standard videotape recording of mental state. Magnetic resonance imaging is also necessary prior to surgery.

\section{Postoperative management}

Physical recovery from surgery is rapid (within a few days). The standardised assessments of mental state can be repeated at this stage and the

Box 6. Who should carry out neurosurgery for mental disorder?

A few specialist centres each with a multidisciplinary team

Multi-disciplinary assessments: psychiatrist, neurosurgeon experienced in stereotactic surgery, clinical psychologist, other relevant members of the mental health care team

Pre-operative management

Evaluate chronicity and lack of response to treatment using standard protocol of the total range of suitable biological and psychological treatments

Obtain informed consent: information sheets are useful in this respect; other relevant family members should also be consulted, but consent must be from patient

Peer review by relevant mental health act or mental welfare commission

Postoperative management

Postoperative rehabilitation programme essential rehabilitation programme initiated. Patients are actively encouraged to a follow a graded programme of occupational and social activities. An important aspect is that there is often a slow response to the operation. Although some patients manifest a dramatic improvement shortly after the operation, the majority pursue a gradual and varying rate of recovery over a period of up to 6-12 months after surgery. It is essential, therefore, to organise a continuing active rehabilitation programme for all patients, which should continue after return to their base hospitals 10 days to two weeks after surgery.

Paradoxically, the significant improvements in mood and mental state following surgery lead to increased independence and assertiveness and place strains on the family relationships, especially when family members are accustomed to having a chronically depressed and therefore submissive quiet and dependent person to care for. Such changes can be challenging for family and friends to adjust to. Equally, the patient may have difficulties in adjusting to independent living after having been used to years of dependence upon other people. Such issues need to be identified by the mental health team responsible for the patient's long-term after-care, and dealt with.

Another factor to take into account is that relapses in mental state occur months and/or years following surgery, even in a patient with a relatively successful outcome. These need to be treated effectively by conventional methods. The patient needs to be reassured that such lapses do not necessarily mean a return to the chronicity of the pre-operative condition. Indeed, it is worthy of comment that patients after surgery often respond better to physical treatments such as electroconvulsive therapy and antidepressant drugs, as well as psychological treatments such as cognitive therapy.

\section{Conclusion}

Just over 60 years after its introduction, psychosurgery in modern dress known as neurosurgery for mental disorder continues to have a role in the management of chronic, treatment-refractory, major depressive and obsessive-compulsive disorders (Sachdev \& Sachdev, 1997). Its practice needs to be restricted to a few specialist centres with rigorous pre-operative assessment and outcome followup, and multi-centre collaboration to facilitate comparative outcome studies of the effects of the different stereotactic lesions. Its application is likely to become even more restricted as advances in other psychiatric treatments progress. It is equally likely that in time a better understanding of the neurobiology of major depressive and obsessive-compulsive 
disorders and the accumulation of 'harder' outcome data will give neurosurgery for mental disorder a stronger theoretical and empirical base. Finally, neurosurgery for mental disorder in the future may not involve lesions in brain tissue. Chronic electrical stimulation through electrodes in the appropriate sites may prove a viable form of treatment similar to that already available for Parkinson's disease. In the longer term, transplantation into the human brain may become a possible treatment strategy.

\section{References}

Anonymous (1941) A prefrontal leucotomy. Lancet, ii, 13

Baer, L., Rauch, S. L., Ballantine, H. T., et al (1995) Cingulotomy for intractible obsessive-compulsive disorder: a prospective long term follow up of 18 patients. Archives of General Psychiatry, 52, 384-392.

Berrios, G. E. (1997) The origins of psychosurgery: Shaw, Burckhardt and Moniz. History of Psychiatry, 8, 61-81.

Bird, J. M. \& Crow, C. D. (1990) Psychosurgery in obsessivecompulsive disorder. In Obsessive-Compulsive Disorder. (eds S. A. Montgomery, W. K. Goodman \& N. Goeting), pp. 82-92. London: Duphar Medical.

Bridges, P. K., Bartlett, J. R., Hale, A. S., et al (1994) Psychosurgery: stereotactic subcaudate tractotomy. An indispensable treatment. British Journal of Psychiatry, 165, 599-611.

CRAG Working Group on Mental Illness (1996) Neurosurgery for Mental Disorder. A report by a Good Practice Group of the CRAG Working Group on Mental Illness. Edinburgh: HMSO.

Crow, H. J. (1961) Controlled multifocal frontal leucotomy for psychiatric illness. Journal of Neurology, Neurosurgery and Psychiatry, 24, 353-360.

Fleming, G. W. T. H. \& McKissock, W. (1943) Prefrontal leucotomy: a further contribution. Lancet, ii, 361-362.

Freeman, W. J., Watts, J. \& Robinson, M. F. (1950) Psychosurgery. In The Treatment of Mental Disorders and Intractible Pain (2nd edn). Springfield, IL: Charles C. Thomas.

Fulton, J. F. (1951) Frontal Lobotomy and Affect: A Neurophysiological Analysis. New York: W. W. Norton.

Hay, P., Sachdev, P., Cumming, S., et al (1993) Treatment of obsessive-compulsive disorder by psychosurgery. Acta Psychiatrica Scandanavica, 87, 197-202.

Hutton, E. L. (1943) Results of prefrontal leucotomy. Lancet, ii, 362-366.

-, Fleming, G. W. T. H. \& Fox, E. E. (1941) Early results of prefrontal leucotomy. Lancet, ii, 3-7.

Hussain, E. S., Freeman, H. \& Jones, R. A. C. (1988) A cohort study of psychosurgery cases from a defined population. Journal of Neurosurgery, Neurology and Psychiatry, 51, 345352.

Kartsouris L. D., Poynton, A., Bridges, P. K., et al (1991) Neuropsychological correlates of stereotactic subcaudate tractotomy: a prospective study. Brain, 114, 2657-2673.

Kiloh, L. G., Smith, J. S. \& Johnson G. F. (1988) Physical Treatments in Psychiatry, pp. 277-333. Melbourne: Blackwel Science.

Kitchen, N. (1995) Neurosurgery for affective disorders at Atkinson Morley's Hospital 1948-1994. Acta Neurochirurgica Supplementum (Wien), 64, 64-68.

Mindus, P. \& Nyman, H. (1991) Normalization of personality characteristics in patients with incapacitating anxiety disorders after capsulotomy. Acta Psychiatrica Scandanavica, 83, 282-291.

-, Rasmussen, S. A. \& Lindquist, C. (1994) Neurosurgical treatment for refractory obsessive-compulsive disorder: implications for understanding frontal lobe function. Journal of Neuropsychiatry and Clinical Neuroscience, 6, 467-477.

-, Edman, G. \& Andréewitch, S. (1999) A prospective long-term study of personality traits in patients with intractible obsessional illness treated by capsulotomy. Acta Psychiatrica Scandinavica, 99, 40-50.

Moniz, E. (1936) Tentatives Operatories dans le Traitement de Certaines Psychoses. Paris: Masson.

National Commission for the Protection of Human Subjects of Biomedical and Behavioral Research (1977) Report and Recommendations: Psychosurgery. Department of Health, Education and Welfare Publication No. (OS) 77-0002. Washington: US Government Printing Office.

Pippard, J. (1955) Rostral leucotomy: a report on 250 cases personally followed up after 1.5-5 years. Journal of Mental Science, 101, 756-773.

Post, F., Linford-Rees, W. \& Schurr, P. H. (1968) An evaluation of bimedial leucotomy. British Journal of Psychiatry, 114, 1223-46.

Pressman, J. D. (1998) Last Resort. Psychosurgery and the Limits of Medicine. Cambridge: Cambridge University Press.

Sachdev, P. \& Hay, P. (1995) Does neurosurgery for obsessivecompulsive disorder produce personality change? Journal of Nervous and Mental Disorder, 183, 408-413.

Sachdev, P. \& Sachdev, J. (1997) Sixty years of psychosurgery: its present status and its future. Australian and New Zealand Journal of Psychiatry, 31, 457-464.

Shutts, D. (1982) Lobotomy. Resort to the Knife. New York: Van Nostrand Reinhold.

Snaith, R. P. (1994) Psychosurgery: controversy and enquiry. British Journal of Psychiatry, 165, 582-584.

Swayze, V. W. (1995) Frontal leukotomy and related psychosurgical procedures in the era before antipsychotics (1935-1954): a historical review. American Journal of Psychiatry, 152, 505-515.

Tooth, G. C. \& Newton, M. P. (1961) Leucotomy in England and Wales 1942-1954. Reports on Public Health and Medical Subjects No. 104. London: HMSO.

World Health Organization (1976) Health Aspects of Human Rights: With Special Reference to Developments in Biology and Medicine. Geneva: WHO.

\section{Multiple choice questions}

1. The following list of people pioneered the operation linked to their name:

a Walter Freeman limbic leucotomy

b Almeida Lima transorbital lobotomy

c Geoffrey Knight subcaudate tractotomy

d John Fulton amygdalotomy

e Wylie McKissock anterior temporal lobectomy

2. Contemporary neurosurgery for mental disorder includes the following operations:

a anterior temporal lobectomy

b bilateral anterior cingulotomy

c amygdalotomy

d sucaudate tractotomy

e transorbital lobotomy

3. Indications for neurosurgery for mental disorder include:

a treatment refractory major depressive disorder

b treatment refractory obsessive-compulsive disorder

c schizoaffective disorder

d complex partial seizures

e Gilles de la Tourette syndrome 
4. Essential preparation for neurosurgery for mental disorder includes the following:

a demonstration of chronicity of the target disorder

b the use of a treatment protocol to show failure to respond to all available standard treatments

c multi-disciplinary assessment

$\mathrm{d}$ an independent peer review process

e establish patients capacity to give informed consent

5. The following are acute postoperative complications of stereotactic surgery for mental disorder:

a transient incontinence of urine

b shortlived mental confusion c paranoid ideation

d ataxia

e status epilepticus

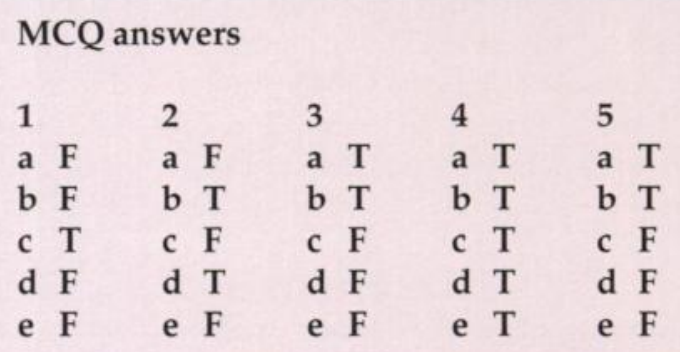

\section{New from Gaskell}

Gaskell is the imprint of the Royal College of Psychiatrists

\section{Late-Onset Mental Disorders}

\section{Edited by Andreas Marneros}

The association between certain diseases and particular periods of life has been studied since the 19th century, yet attempts to delineate categories of mental disorder unique to old age have floundered over the decades, and the debate continues unabated.

After an historical overview, this book looks at differences between early-onset and late-onset disorders. Is there anything special about old-age depression? Are there any atypical features of late-onset schizophrenia? Besides questions concerning depression, dementia and psychosis, the book looks at sleep disturbances in the elderly, anxiety, use of anti-dementia drugs, anti-depressants and neuroleptics in old age, and psychological processes.

It will be of particular interest to old age psychiatrists, liaison psychiatrists, epidemiologists, university lecturers and medical historians.

June, 1999, 208pp, Paperback, ISBN 190124226 9, £25.00

\section{Gaskell books are available from:}

Book Sales, Royal College of Psychiatrists, 17 Belgrave Square, London SW1X 8PG. Telephone +44 (0)171 2352351 ext. 146, fax +44 (0) 171 245 1231. Credit card orders can be taken over the telephone.

See the latest information on College publications on the Internet at: 